\title{
A Suggestion for Modification of Vafa-Witten Theory
}

\author{
Pei Wang \\ Institute of Modern Physics,Northwest University \\ P.O.Box 105,Xian, 710069, China
}

December 10, 2017

\begin{abstract}
Using the Mathai-Quillen formalism we reexamine the twisted $N=4$ supersymmetric model of Vafa-Witten theory. Smooth out the relation between the supersymmetric action and the path integral representation of the Thom class.
\end{abstract}

IMP-NWU-951201 


\section{Introduction}

Recently Edward Witten made a breakthrough on the Donaldson theory by his magical monopole equation [1]. He made also important developments with N.Seiberg and C.Vafa $[2,3,4]$ in his topological field theory[5]. These developments enrich the Donaldson theory enormously, so that it can be explicitly described by using of quantum field theories in variours supersymmetric models. In reference [4] the partition functions of a twisted $N=4$ supersymmetric Yang-Mills theory were computed on certain 4-manifolds and shown to satisfy the remarkable Montonen-Olive "S-duality conjectures". One important part of this story is that this model express the partition function as an Euler characteristic of the moduli space of instantons.

However, it seems to us that two things might be improved. The first is in their paper, the gauge transformation parameter, field $\Phi$, is independent of the curvature derived from the connection on a vector bundle with instanton moduli space (precisely, moduli space dividing by gauge group) as its base space. We know that this connection is defined through the action of gauge group[6,7]. There must be close relation between them. The second is that the connection we refer to is an object, which is hard to generalize to a superfield. Of couse, there would be two ways to treat this object. One was to consider it as a functional of a superfield. The other was thinking of it as a component of a supermultiplets, which can be made of a new superfield. But both lead to strange gauge transformation rules and great complications. It is pleasure to smooth these problems.

In this paper, we would like to assume that the gauge parameter $\Phi$ is identified with the "curvature" (we will give the exact meaning of it in the section 3 ) on the bundle with instanton base space. By using of the Mathai-Quillen formula we develop the partition function of Vafa-Witten theory in the Cartan model[8,9,10]. The result is equal to a Yamron's action (with a missing term added)[11]. Under suitable vanishing theorems, which were discussed carefully by Vafa and Witten, this partition function is expressed by an Euler characteristic (equivalently, a Thom class) strictly.

\section{Mathai-Quillen formulae of Thom class}

Since Witten and his colleagues have expressed a partition function (more generally, correlation function) of topological field as the Euler characteristic, it is quite nature that the celebrated Mathai-Quillen formulae of Thom class[8] are very powerful tools[10.12]. Let us recall something related. Let $E$ be an oriented real vector bundle of rank $2 m$, with base space $M$ and standard fiber $V$, and

$$
s: M \rightarrow E
$$


be the zero section. The Euler class can be obtained through the pull back of map $S$ from Thom class $\Phi(E)$,

$$
e(E)=s^{*} \Phi(E)
$$

Mathai and Quillen found an explicit formula for the universal Thom class $U[8,10]$. The criteria are 1) basic. 2) closed, 3) the integration along the fiber is equal to unity, $\int_{V} U=1$. The last criteron is connected with the definition of Thom class [13]: the inverse of push forward of $1 \in H^{0}(M)$ is defined as Thom class, $\pi_{*}^{-1}=\Phi(E) \in H_{V R D}^{2 m}(E)$, in which "VRD" denotes that "rapid decrease along fiber", which is equivalent to the "vertical compact supports". The formulae make use of equivariant cohomology in which the Weil algebra plays the part of the universal bundle. In terms of generators the Weil algbra $W(g)$ is expressed as follows[14]

$$
\begin{array}{r}
d_{w} \theta=\phi-\theta^{2}, \quad d_{w} \phi=\phi \theta-\theta \phi, \\
\theta=T_{i} \theta^{i}, \quad \phi=T_{i} \phi^{i}, \quad T_{i} \in S O(2 m) .
\end{array}
$$

Through the Chern-Weil homomorphism (the basic form see for example [8] or [10])

$$
(W(g) \otimes \Omega(V))_{b a s i c} \equiv \Omega_{G}(V) \rightarrow \Omega(E),
$$

we get the real differential form on the vector bundle -partition function of the moduli space.

There are three expressions for the universal Thom class. The first two are $[8,10]$

$$
U=\frac{1}{(2 \pi)^{m}} P f a f f i a n(\phi) e^{-\frac{1}{2 \lambda}<\chi, \chi>-\frac{1}{2 \lambda}<\nabla \chi, \phi^{-1} \nabla \chi>}
$$

and

$$
U=\frac{\lambda^{m}}{(2 \pi)^{m}} e^{-\frac{1}{2 \lambda}<\chi, \chi>} \int d \rho \exp \left\{\frac{1}{2 \lambda}<\rho, \phi \rho>+\frac{i}{\lambda}<\nabla \chi, \rho>\right\}
$$

in which inner product is defined on $V, \rho$ is an antighost variable, and

$$
\nabla \chi=d x+\theta \chi
$$

Following Vafa and Witten we also introduce a parameter $\lambda$ in the formulae. From these two expressions criteria 1) and 3) are easily proved[8,10]. (The integration representation (3) derives from (2) through a Gauss integration by use of $\phi^{T}=-\phi$ ). To check the criterion 2) so that we can write down the partition function of the model later, we need the third expression. 
As usual we introduce again a zero ghost number field $\pi$ to complete the enlarged equivariant cohomology complex $W(g) \otimes \Omega(V) \otimes \Omega\left(\Pi V^{*}\right)$ (the $\Pi$ signifies that the coordinates are to be regarded as anticommuting, see [10]). But, instead of the common used deRham differential $\delta \rho=\pi, \delta \pi=0[10]$ we use the Vafa-Witten mechanism. Let $\delta=d_{w}$ for Weil algebra, and

$$
\begin{array}{r}
\delta \rho=\pi-\theta \rho, \\
\delta \pi=-\theta \pi+\phi \rho .
\end{array}
$$

It is easy to prove from (1) and (5) that $\delta^{2}=0$. Let $Q=d+\delta, d$ is the exterior differential on $\Omega(V)$, we then have the third formula

$$
U=\frac{1}{(2 \pi)^{2 m}} \int_{V^{*} \times \Pi V^{*}} \prod_{m=1}^{2 m} d \pi_{n} d \rho_{n} e^{[Q, \Psi]}
$$

in which the gauge fermion is

$$
\Psi=\frac{1}{2 \lambda} \rho(2 i \chi-\pi)
$$

The close of Thom form $U$ follows from the following observation:

$$
(d+d w) \int(\cdots)=\int[Q,(\cdots)]=0
$$

When $U$ is transferred to a partition function of a real field model through the ChernWeil homomorphism, the generators of Weil algebra will correspond the connection $\Theta$ and curvature $\Phi$ on the vector bundle of moduli space respectively:

$$
\theta \rightarrow \Theta, \quad \phi \rightarrow \Phi
$$

\section{Gauge invariance and Cartan model}

Now, let us consider the case $\delta^{2} \neq 0$, corresponding a gauge transformation. Let $\mathcal{U}$ denotes the priciple bundle consisting of Yang-Mills potentials. Locally it can be parametrized by

$$
A=g^{-1} a g+g^{-1} d g, \quad a \in \operatorname{orbit} \mathcal{U} / \mathcal{G}, \quad g \in \text { gauge group } \mathcal{G} .
$$

It gives $(\delta d+d \delta=0)$

$$
\delta A=g^{-1} \delta a g-D_{A}\left(g^{-1} \delta g\right)
$$


where

$$
D_{A} \Lambda=d \Lambda+A \Lambda-(-)^{p} \Lambda A,
$$

$p$ is the parity of $\Lambda$ (counting of both $d$ and $\delta$ ). A connection can be introduced on $\mathcal{U}[6]$.

$$
\Theta=-G_{A} D_{A}^{\dagger} \delta A, \quad G_{A}=\left(D_{A}^{\dagger} D_{A}\right)^{-1},
$$

such that

$$
\left.\Theta\right|_{\text {fiber }}=g^{-1} \delta g .
$$

Up to a zero mode of $D_{A}^{\dagger}$ we may write

$$
\delta A=-D_{A} \Theta .
$$

From this connection a formal "curvature" is derived as

$$
\Phi=\delta \Theta+\Theta^{2},\left.\quad \Phi\right|_{\text {fiber }}=g^{-1} \delta^{2} g .
$$

We can define the local gauge transformation in case at hand,

$$
\delta_{G}=\epsilon \delta^{2}
$$

wher $\epsilon$ is a Grassmann number anticommuting with $d$ and $\delta$, so that

$$
\delta_{G} A=g^{-1} \delta_{G} a g-D_{A}\left(g^{-1} \delta_{G} g\right) .
$$

And eq.(15) leads to

$$
\delta_{G} A=-D_{A} \epsilon \Phi=\epsilon D_{A} \Phi .
$$

However, this $\Phi$ does not transform like a curvature under a "bundle gauge transformation" $\Theta \rightarrow \Theta^{\prime}=h^{-1} \Theta h+h^{-1} \delta h$. So we have to define a real curvature $\Omega$ as follows. Let $\Theta=\delta A^{i} \Theta_{i}$, then we have

$$
\Phi=\delta^{2} A^{i} \Theta_{i}+\Omega, \quad \Omega=\frac{1}{2} \delta A^{i} \delta A^{j} \Omega_{i j} .
$$

Any way we may have

$$
\delta A=\Psi, \quad \delta \Psi=D_{A} \Phi .
$$


If we again use the correspondence between Weil algebra and connection as well as curvature (9), the Weil algebra must change to

$$
d_{w}^{\prime} \theta=\phi-\theta^{2}, \quad d_{w}^{\prime}=d_{w}^{\prime 2} \theta^{2}+\phi \theta-\theta \phi .
$$

Changing eq.(5) also to (see [4])

$$
\begin{array}{r}
\delta \rho=\pi-\theta \rho, \\
\delta \pi=-\theta \pi+\Omega \rho,
\end{array}
$$

we find that

$$
[Q, \Psi]=-\frac{1}{2 \lambda}(\pi-i \chi)^{2}-\frac{1}{2 \lambda}<\chi, \chi>+\frac{1}{2 \lambda}<\rho, \Omega \rho>+\frac{i}{\lambda}<\nabla \chi, \rho>.
$$

So we have the same expressions in the first two formulae for Thom class (2) and (3), with $\Omega$ instead of $\Phi$ only. And the criteria 1) and 3) for Thom form are satisfied. To require that the form is closed, the gauge fermion $\Psi$ must be gauge invariant, i.e. $\{Q[Q, \Psi]\}=0$. As a matter of fact, however, we find that

$$
\left.\{Q[Q, \Psi]\}=-\frac{1}{2 \lambda}\left\{2 i \rho(\Omega-\Phi) \chi+\delta^{2} A^{i} \delta A^{j}\right) \rho \Omega_{i j} \rho\right\} \neq 0 .
$$

To overcome this difficult we have to choose the Cartan model $S\left(g^{*}\right) \otimes \Omega(V)$,

$$
\theta \rightarrow 0, \quad d \rightarrow d_{c}=d-i_{\phi}
$$

where $i_{\phi}$ is the contraction operator $[8,9,10]$. In the case at hand, $\Omega$ is undistinguishable from $\Phi$. Let $\delta=d_{c}$ on $\Omega(V)$, and

$$
\begin{gathered}
\delta \rho=\pi, \\
\delta \pi=\phi \rho .
\end{gathered}
$$

It is easy to check that

$$
\delta \Psi=\left[Q_{c}, \Psi\right]=-\frac{1}{2 \lambda}(\pi-i \chi)^{2}-\frac{1}{2 \lambda}<\chi, \chi>+\frac{1}{2 \lambda}<\rho, \phi \rho>+\frac{i}{\lambda}<d \chi, \rho>.
$$

Keeping correct the properties 1 ) and 3 ), it is also clear that

$$
\left\{Q_{c}\left[Q_{c}, \Psi\right]\right\}=0
$$

so that

$$
U=\frac{1}{(2 \pi)^{m}} \int_{V^{*} \times \Pi V^{*}} \prod_{n=1}^{2 m} d \rho_{n} d \pi_{n} e^{\left[Q_{c}, \Psi\right]}
$$

is closed. Cartan model is also a favorable matter, because of that to construct Donaldson invariants from quantum field theory we need $[5,2,12]$

$$
\delta \phi=0 \text {. }
$$




\section{Projection by the gauge freedom}

When we treat the topological Yang-Mills theory, the gauge invariance must be considered. The moduli space should be divided by $G=G_{\text {gauge }}$, and in fact we will compute the Euler characteristic of the quotient bundle $E / G$. This can be done by introducing two new fields $\bar{\phi}, \eta$ of ghost number -2 and -1 respectively. And let

$$
\begin{array}{r}
\delta \bar{\phi}=\left[Q_{c}, \bar{\phi}\right]=\eta, \\
\delta \eta=\left[Q_{c}, \eta\right]=[\bar{\phi}, \phi] .
\end{array}
$$

The consequence of the gauge invariance leads to a projection gauge fermion[10]

$$
\Psi_{\text {proj }}=\psi D \bar{\phi}
$$

where $\psi$ is defined in eq. (21). The Thom class of interest becomes the following form $(t=\operatorname{dim} G)$

$$
\begin{aligned}
\Phi(E / G) & =\frac{1}{(2 \pi)^{m+t} i^{t}} \int \prod d \bar{\phi} d \eta d \rho d \pi e^{\left[Q_{c}, \Psi_{t o t}\right]} \\
\Psi_{\text {tot }} & =\Psi_{\text {local }}+\Psi_{\text {proj. }}+\Psi_{n o n .}
\end{aligned}
$$

in which $\Psi_{\text {local }}=\Psi$ is the localized gauge fermion $(7)$ obtained earlier, and $\Psi_{n o n}$. is a possible nonminimal term[4].

\section{$5 \quad N=4$ twisted supersymmetric Yang-Mills model}

Now we are in the position to construct the concret model. Let $\mathcal{M}$ denote the moduli space of antiselfdual instantons, $\mathcal{V}$ denote the fiber, what we are interested in is the vector bundle $\varepsilon=\mathcal{M} \times{ }_{\mathcal{G}} \mathcal{V}$. Through out the Chern-Weil homomorphism we will introduce various fields to realize the Thom form, the partition function. Before that, as is pointed out by Vafa and Witten, when the parameter $\lambda$ approaches to zero the integral leads to a trivial results but with signs, a standard theorem that the Euler class of a bundle is computed by counting the zeros of a section, weighted with signs. To avoid the cancellation of Euler class due to different signs they invent a way of doubling the number of fields.

Therefore we have all of the twisted $N=4$ supersymmetric fields[4] (The ghost numbers are marked in the brackets):

$$
\begin{array}{ll}
A_{i}, B_{i j}(0) & \text { coordinates of moduli space; } \\
\psi_{i}, \tilde{\psi}_{i j}(1) & \text { tangent to the moduli manifold, }
\end{array}
$$




$$
\begin{aligned}
& \chi_{i j}, \tilde{\chi}_{i}(-1) \text { for } \rho, \text { coordinates of fiber; } \\
& H_{i j}, \tilde{H}_{i}(0) \quad \text { for } \pi, \text { auxiliary fields, the super } \\
& \text { partners of } \chi_{\mathrm{ij}}, \tilde{\chi}_{\mathrm{i}} \text { respectively; } \\
& \phi(2), \tilde{\phi}(-2), \eta(-1) \quad \text { associated with the symmetry group } G, \\
& \text { the same fields we have used; } \\
& C(0), \xi(1) \quad \text { associated with the symmetries, } \\
& \text { reprsenting the horizontal projection, }
\end{aligned}
$$

the super partners of $A_{i}, B_{i j}$ respectively;

in which the supermultiplet $\left(A_{i}, \psi_{i}\right)$ have presented in eq.(21). Moreover, we have also to substitute the coordinates $\chi$ in the Thom form by the section pairs[4]

$$
S_{i j}=F_{i j}^{\dagger}+\frac{i}{2}\left[C, B_{i j}^{\dagger}\right]+\alpha\left[B_{k i}^{\dagger}, B_{j}^{\dagger k}\right]
$$

and

$$
k_{i}=2 D^{j} B_{i j}^{\dagger}+\frac{1}{2 i} D_{i} C
$$

in which $\alpha$ is a constant, $F_{i j}^{\dagger}$ and $B_{i j}^{\dagger}$ are antiself-dual part of $F_{i j}=\partial_{i} A_{j}-\partial_{j} A_{i}+\left[A_{i}, A_{j}\right]$ and $B_{i j}$ ( we will omit the symbol $\dagger$ for $B_{i j}^{\dagger}$ for simplicity). Noting that the projection gauge fermion needs also doubled we obtain the partition function of the model as follows

$$
Z=N \int[d \Sigma] \exp \left\{Q_{c}\left(\Psi_{\text {local }}+\Psi_{\text {proj. }}+\Psi_{\text {non. }}\right\}\right.
$$

where $[d \Sigma]$ is the measure consisting of all of the thirteen fields, $N$ is the normalization constant,

$$
\begin{aligned}
\Psi_{\text {local }}= & T_{r}\left\{\chi ^ { i j } \left(2 i F_{i j}^{\dagger}-\left[C, B_{i j}\right]+2 i \alpha\left[B_{k i}, B_{j}{ }^{k}\right]-H_{i j}\right.\right. \\
& \left.+\tilde{\chi}^{i}\left(4 i D^{j} B_{i j}+D_{i} C-\tilde{H}_{i}\right)\right\}
\end{aligned}
$$

and

$$
\Psi_{\text {proj }}=-T_{r}\left\{\psi^{i} D_{i} \tilde{\phi}+\tilde{\psi}^{i j}\left[B_{i j}, \tilde{\phi}\right]\right\}
$$

one may also have

$$
\Psi_{\text {non }}=\beta T_{r}\{\eta[\tilde{\phi}, \phi]+2 C[\tilde{\phi}, \xi]\}, \beta=\text { const } .
$$

It is clear that if there are suitable vanishing theorems as was discussed carefully by Vafa and Witten,i.e. $B_{i j}=C=0[4]$, set $\beta=0$ the partition function identifies a Thom class (equivalently, an Euler characteristic). 


\section{$6 \quad$ BRST and anti BRST transformations}

One more point we would like to explain is that the twisted $N=4$ system has $N=$ 2 topological symmetry and an $S U(2)$ symmetry. We have not only a $Q_{1} \equiv Q_{c}$, but $Q_{A} \quad A=1,2$ pair. One is thought of a BRST operator, the other can be identified with an anti BRST charge[11]. Let us introduce following superfields:

$$
T^{i}=\partial^{i}+A^{i}+\theta^{A} \psi_{A}^{i}+\frac{\epsilon_{A B} \theta^{A} \theta^{B}}{2} \tilde{H}^{i}
$$

in which $\psi_{1}^{i}=\psi^{i}, \psi_{2}^{i}=\tilde{\chi}^{i}$;

$$
Y^{i j}=B^{i j}+\theta^{A} \chi_{A}^{i j}+\frac{\epsilon_{A B} \theta^{A} \theta^{B}}{2} H^{i j}
$$

in which $\chi_{1}^{i j}=\tilde{\psi}^{i j}, \chi_{2}^{i j}=\chi^{i j}$

$$
X_{A B}=\phi_{A B}+\frac{1}{2}\left(\theta_{A} \eta_{B}+\theta_{B} \eta_{A}\right)-\frac{\epsilon_{C D} \theta^{C} \theta^{D}}{4}\left[\phi_{A E}, \phi_{B}^{E}\right]
$$

in which $\phi_{11}=\phi^{22}=\phi, \phi_{22}=\phi^{11}=\tilde{\phi}$, and $\phi_{12}=\phi_{21}=-\phi^{12}=-\phi^{21}=C$. The supersymmetry transformations are determined by

$$
Q_{A}=\frac{\partial}{\partial \theta^{A}}+\theta^{B}\left[\phi_{A B}, \cdot\right]+\frac{\epsilon_{B C} \theta^{B} \theta^{C}}{4}\left[\eta_{A}, \cdot\right]
$$

in which we add a two $\theta$ term so that the three transformation rules derived from the

superfield $X_{A B}$ are selfconsistant. And it also gives the same transformation laws as Yamron's[11]. In fact, the transformation laws may write as

$$
\delta S=\epsilon^{A}\left[Q_{A}, S\right], \quad S=T^{i}, Y^{i j}, X_{A B} .
$$

More detail we get

$$
\begin{aligned}
\delta A^{i} & =\epsilon^{1} \psi^{i}+\epsilon^{2} \tilde{\chi}^{i} \\
\delta \psi^{i} & =\epsilon^{1} D^{i} \phi-\epsilon^{2}\left(\tilde{H}^{i}-D^{i} C\right) \\
\delta \tilde{\chi}^{i} & =\epsilon^{1}\left(\tilde{H}^{i}+D^{i} C\right)+\epsilon^{2} D^{i} \tilde{\phi} \\
\delta \tilde{H}^{i} & =\epsilon^{1}\left(\left[C, \psi^{i}\right]-\left[\phi, \tilde{\chi}^{i}\right]-\frac{1}{2} D^{i} \xi\right)+\epsilon^{2}\left(\left[\tilde{\phi}, \psi^{i}\right]-\left[C, \tilde{\chi}^{i}\right]-\frac{1}{2} D^{i} \eta\right), \\
\delta B^{i j} & =\epsilon^{1} \tilde{\psi}^{i j}+\epsilon^{2} \chi^{i j} \\
\delta \tilde{\psi}^{i j} & =\epsilon^{1}\left[B^{i j}, \phi\right]-\epsilon^{2}\left(H^{i j}+\left[C, B^{i j}\right]\right),
\end{aligned}
$$




$$
\begin{aligned}
\delta \chi^{i j}= & \epsilon^{1}\left(H^{i j}-\left[C, B^{i j}\right]\right)-\epsilon^{2}\left[\tilde{\phi}, B^{i j}\right], \\
\delta H^{i j}= & \epsilon^{1}\left(\left[C, \tilde{\psi}^{i j}\right]-\left[\phi, \chi^{i j}\right]+\frac{1}{2}\left[\xi, B^{i j}\right]\right) \\
& +\epsilon^{2}\left(\left[\tilde{\phi}, \tilde{\psi}^{i j}\right]-\left[C, \chi^{i j}\right]+\frac{1}{2}\left[\eta, B^{i j}\right]\right), \\
\delta \phi= & -\epsilon^{2} \xi \\
\delta C= & \frac{1}{2}\left(\epsilon^{1} \xi-\epsilon^{2} \eta\right) \\
\delta \tilde{\phi}= & \epsilon^{1} \eta, \\
\delta \eta= & \epsilon^{1}[\tilde{\phi}, \phi]+2 \epsilon^{2}[\tilde{\phi}, C], \\
\delta \xi= & 2 \epsilon^{1}[C, \phi]+\epsilon^{2}[\tilde{\phi}, \phi] .
\end{aligned}
$$

For the parts proportional to $\epsilon^{1}$ we find the transformation laws we used before. Using the anti-BRST transformations (proportional to $\epsilon^{2}$ ) we can prove that

$$
\begin{aligned}
\Psi_{t o t}= & Q_{2} W \\
W= & T_{r}\left\{B^{i j}\left(2 i F_{i j}-H_{i j}\right)+\frac{2 i \alpha}{3} B_{i j}\left[B_{k}{ }^{i}, B^{j k}\right]\right. \\
& \left.+\psi^{i} \tilde{\chi}_{i}+\beta \phi_{A B}\left[\phi_{C}{ }^{A}, \phi^{B C}\right]\right\} .
\end{aligned}
$$

In conclusion, we have

$$
Z=N \int[d \Sigma] e^{\frac{1}{2}\left\{Q^{A}\left[Q_{A}, W\right]\right\}}
$$

It agrees with the action obtained by Yamron except an added term $B_{i j}\left[B_{k}{ }^{i}, B^{j k}\right]$.

\section{Summary}

To sum up, we have derived the partition function of the twisted $N=4$ supersymmetric Yang-Mills theory, and it reduces to the Euler characteristic under suitable vanishing theorems. We can also integrate over auxiliary fields, the main part of the result have already presented in Vafa-Witten's paper[4], i.e. $|s|^{2}+|k|^{2}$. One interesting thing is to find the corresponding formulae in the Weil model. This relats to that how to combine a basic form with the gauge transformation, or how to find a gauge invariant gauge fermion. We have not found the answer yet. 


\section{Acknowlegements}

The author would like to thank Ke Wu for hospitality at Institute of Theoretic Physics in Beijing, and benefit discussions, especially drawing his interest to the Mathai-Quillen formalism. He also would like to thank Yi-hong Gao for valuable introduction talk about Donaldson-Witten theory and bringing him the preprint[10]. The work is supported in part by the National Science Fund of China.

\section{References}

[1] E.Witten, "Monopoles and four-manifolds", preprint IASSNS-HEP-94-96, hepth/9111102,1994

[2] W.Witten, J.Math.Phys.35(1994)5101.

[3] N.Seiberg and E.Witten, Nucl.Phys.B426 (1994)19;Nucl.Phys.B431(1994)484.

[4] C.Vafa and E.Witten, Nucl.Phys.B431(1994)3.

[5] E.Witten, Comm.Math.Phys.117(1988)353.

[6] M.F.Atiyah and I.M.Singer, Proc.Natl.Acad.Sci. U.S.A.81(1984)2597;R.Stora, Cargese Lectures 1983 Annecy preprint LAPP-TH-94.

[7] M.F.Atiyah and L.Jeffrey, J.Geom.Phys.7(1990)119.

[8] V.Mathai and D.Quillen, Topology 25(1986)85.

[9] J.Kalkman, Comm.Math.Phys.153(1993)447.

[10] S.Cordes, G.Moore and S.Rangoolam, preprint "Lectures on 2D Yang-Mills Theory, Equivariant Cohomology and Topological Field Theories" hep-th/9411210, YCTPP11-94,1994.

[11] J.Yamron, Phys.Lett.B213(1988)325.

[12] J.M.F.Labastida and M.Marino," A Topological Lagrangian for Monopoles on Fourmanifolds." preprint US-FT-3/93, hep-th@xxx/yymmddd.1995.

[13] R.Bott and L.Tu, Differential Forms in Algebraic Topology, Springer Verlag, New York,1982.

[14] M.F.Atiyah and R.Bott, Topology 23(1984)1. 